Case Report

\title{
Recurrent dermatofibrosarcoma protuberans: A Case report and literature review
}

Hari Bahadur KC, Jayan Man Shrestha, Ishwar Lohani

\section{Abstract}

Introduction: Dermatofibrosarcoma protuberans is a rare soft tissue tumor which is known for its high recurrence behavior. Adequate excision and regular follow up is the current treatment modality.

Case report: 42 years male developed multiple nodular lesions on anterior chest wall for which he underwent surgical therapy for five times, but tumor recurred on every occasion. Wide local excision and split skin grafting was performed with negative margin on histopathology.

Conclusion: Dermatofibrosarcoma protuberans has high recurrence rate due to its infiltrative growth pattern. Regular follow up is required after surgery during early post-operative period to detect any recurrences.

Keywords: Dermatofibrosarcoma protuberans; Recurrent; Wide local excision.

\section{Author affiliations:}

Department of Plastic Surgery and Burns, Tribhuvan University Teaching Hospital, Kathmandu, Nepal

Correspondence:

Dr. Hari Bahadur KC,

Department of Surgery, Gandaki Medical College Teaching Hospital, Pokhara, Nepal

Email: hari_kc7@yahoo.com

ORCID:

Copyright information:

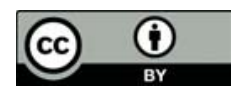

How to cite this article:

KC HB, Shrestha JM, Lohani I. Recurrent dermatofibrosarcoma protuberans: a case report and literature review. J Soc Surg Nep. 2020 Dec;23(2):67-69.

DOI:

https://doi.org/10.3126/jssn.v23i2.35844

\section{Introduction}

Dermatofibrosarcoma protuberans (DFSP) is a relatively uncommon soft tissue neoplasm with intermediate- to lowgrade malignancy. It arises from the dermis and invades deeper subcutaneous tissue (e.g. fat, fascia, muscle, bone). Darier and Ferrand first described it as a distinct cutaneous disease entity called progressive and recurring dermatofibroma and in 1925, Hoffman officially coined the term dermatofibrosarcoma protuberans. ${ }^{1,2}$

It is a slow growing tumor which appears as painless nodule or thickened area of skin (plaque) and size may range from small nodule to several centimeters large tumors. Ulceration and hemorrhage are the late manifestations when the tumor grows into certain size. Although metastasis rarely occurs, DFSP is a locally aggressive tumor with a high recurrence rate. The pigmented variant of DFSP is also known as Bednar tumor, which is very rare and accounts only one percent of all DFSP cases. ${ }^{3}$
Surgical excision remains the mainstay of treatment for DFSP. Wide local excision and Mohs micrographic surgery are the two modalities of surgical therapy. Because of the high recurrence rate, close follow up is required for patients with DFSP after surgical resection.

\section{Case Report}

Forty two years old gentlemen from eastern Nepal presented with a history of multiple swellings over anterior chest wall for the last seven years. The swellings were painless, gradually increasing in size and bled from the larger nodules. He underwent surgery five times in the past for the same swellings but they recurred each time. The last operation was three years back and the tumor recurred after a year. There was no history of cough, chest pain, and anorexia or weight loss. Physical examination revealed multiple nodular masses over anterior chest wall, more on 


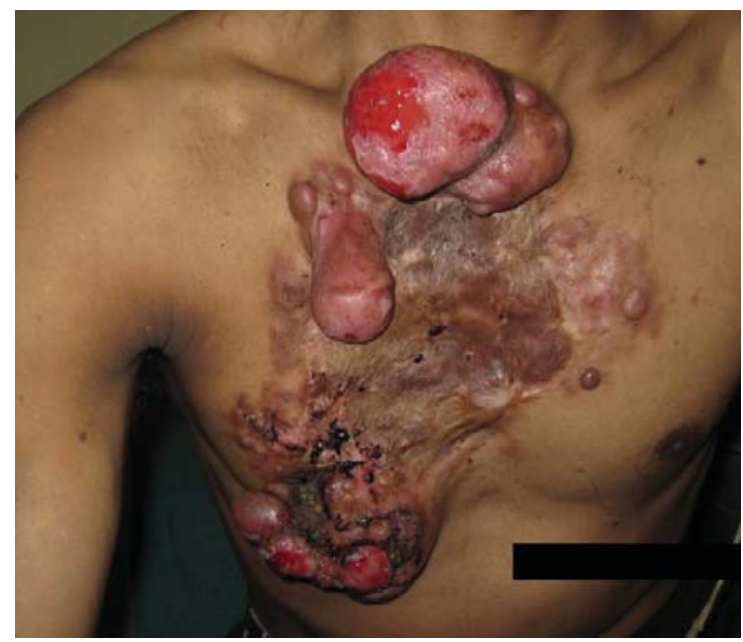

Figure 1. Multiple lobulated nodules over anterior chest wall on the background of previous scar with areas of erosions, hemorrhages and crust formation.

the right side and also extending to the neck (Figure 1). The nodules were non tender, not fixed to deep structures and there was minimal bleeding from the surface erosions. Routine investigations were normal. The tumor was excised with wide local margin of three centimeters, including deep fascia and split skin graft was applied (Figure 2a and 2b). Histopathology report revealed dermatofibrosarcoma protuberance with no lymphovascular invasion and negative resection margins (Figure 3). There was no recurrence till two years follow up.

\section{Discussion}

Dermatofibrosarcoma protuberans (DFSP) is a variant of soft tissue sarcoma accounting for approximately one percent of them. The incidence of DFSP in the United States is 4.2 per million cases per year.4 It usually occurs in adults aged 20-50 years with no significant racial or sexual predominance. The most common site is the trunk (42-72\%), followed by the proximal extremities (16-30\%) and occurrence above the neck is rare.5 The differential diagnosis are dermatofibroma, epidermal inclusion cyst, keloid and hypertrophic scar, malignant melanoma, metastatic carcinoma of the skin, morphea, and some other similar conditions.

A skin biopsy is essential for the definitive diagnosis of DFSP which in most occasions is excisional biopsy. Imaging studies are useful in metastatic diseases, and magnetic resonance imaging (MRI) in particular is good in defining the approximate tumor border and depth of invasion. ${ }^{6}$ A simple staging system published in "Short German guidelines: dermatofibrosarcoma protuberans" may be helpful in clinical use, which divides the tumor into three groups: stage I - primary tumor, localized disease, stage II - lymph node metastasis and stage III - distal metastasis.

Surgical resection is the treatment of choice for DFSP.

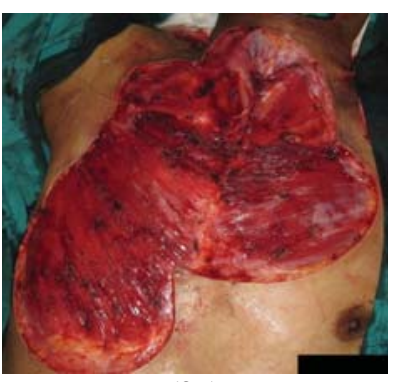

(2a)

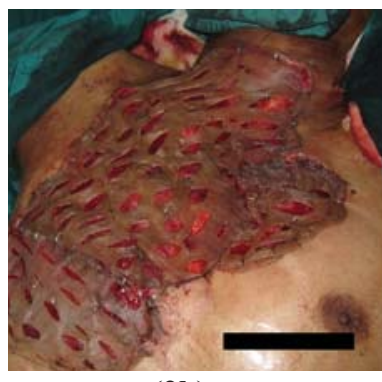

(2b)
Figure 2. Exposed muscles of anterior chest after wide resection of tumor including deep fascia and muscle aponeurosis (2a), wound covered with split skin graft (2b).

Though wide local excision is the widely used method, Mohs micrographic surgery has become the treatment of choice as it requires less tissue removal and allows complete margin assessment. Owing to its infiltrating growth pattern that may extend far beyond the clinical margins; a wide excision of $2-3 \mathrm{~cm}$ or more of the margin including the underlying fascia is recommended. ${ }^{\text {? }}$

Though conventional chemotherapy has no role in DFSP, molecular- targeted therapy with imatinib has been found to be effective especially for unresectable, recurrent or metastatic cases. ${ }^{8}$ Neoadjuvant imatinib therapy for DFSP has been proposed in recent studies. Radiation therapy has had a limited role in DFSP, but it may be used as an adjunct to surgical therapy in situations where resection margins are positive or wide resection results in major cosmetic/ functional deficits. ${ }^{9}$ Postoperative adjuvant radiation therapy may reduce the risk of recurrence when clear surgical margins are not confident.

A local recurrence rate of $10-20 \%$ has been reported by many studies after wide local excision, and approximately half of them recur within two years and $80 \%$ within three years. Hence, patient should be followed up every six monthly during this period and annually thereafter. Poor

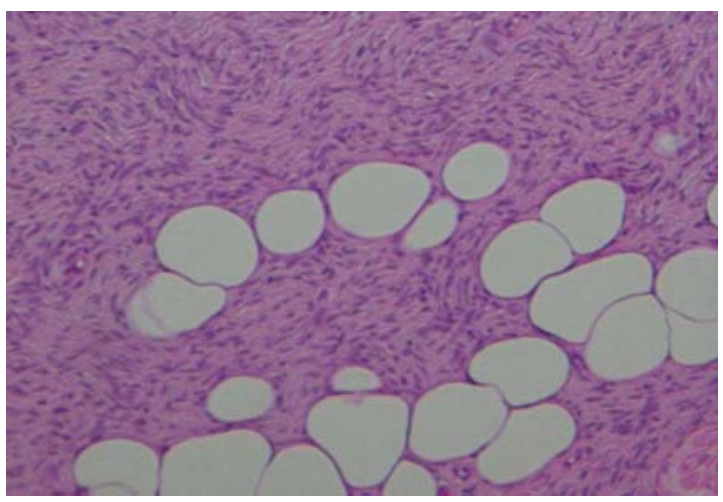

Figure 3. High power view of dermatofibrosarcoma protuberans revealing subcutaneous adipocytes entrapped by densely infiltrative spindle-shaped tumor cells 
prognostic factors are late presentation, aggressive local invasion, regional nodal involvement, distant metastasis and histological features such as DNA aneuploidy, high mitotic figures, and p53 gene mutation. Pooling of data shows a superior cure rate (average recurrence rate of $1.3 \%$ among 463 cases reported vs $20.7 \%$ among 1394 patients treated with wide local excision) when Mohs micrographic surgery is used. Thus, Mohs micrographic surgery is now considered the treatment of choice particularly when a lesion is located in the head and neck region. ${ }^{10}$ However, Mohs surgery is more time consuming, costly and is not readily accessible in many parts of the world.

The present patient had multiple recurrences in the past. Wide surgical excision with three $\mathrm{cm}$ margin at present operation had negative margin for tumor and there was no

\section{References}

1. Darrier J, Ferrand M. Dermatofibromes Progressifs et Recidivants on Fibrosarcomes de la Peau. Ann De Dermat Et Syph 1924;5:545.

2. Hoffmann E. Uber das knollentreibende firbosarkon der haut (dermatofibrosarcoma protuberans). Dermat Ztschr 1925;43:1.

3. Lopez JI, Elizalde JM, Fernandez-Larrinoa A. Pigmented dermatofibrosarcoma protuberans (Bednar tumour). Dermatology 1992;184:281-2.

4. Criscione VD, Weinstock MA. Descriptive epidemiology of dermatofibrosarcoma protuberans in the United States, 1973 to 2002. J Am Acad Dermatol 2007 Jun;56(6):968-73.

5. Rahman GA, Adigun IA, Buhari MO, Ogundipe KO, Omotayo JA. Dermatofibroma protuberans: experience with management of 18 cases. European Journal of Scientific Research 2009;25(1):145-50.

6. Riggs K, McGuigan KL, Morrison WB, Samie FH, Humphreys T. Role of magnetic resonance imaging recurrence till two years follow up. Mohs micrographic surgery is not available at present in our institute.

\section{Conclusion}

Dermatofibrosarcoma protuberans is a relatively uncommon soft tissue neoplasm mostly occurring on the trunk and extremities, and has a high recurrence rate after excision due to its infiltrative growth pattern. While wide local excision is the widely used procedure with good results, Mohs micrographic surgery has been increasingly accepted as the treatment of choice in recent times. Regular post-operative follow up is necessary for early detection and treatment of recurrences.

in perioperative assessment of dermatofibrosarcoma protuberans. Dermatol Surg Dec 2009;35(12):203641.

7. Meguerditchian AN, Wang J, Lema B, Kraybill WG, Zeitouni NC, Kane JM 3rd. Wide excision or Mohs micrographic surgery for the treatment of primary dermatofibrosarcoma protuberans. Am J Clin Oncol Jun 2010;33(3):300-3.

8. McArthur G. Molecularly targeted treatment for dermatofibrosarcoma protuberans. Semin Oncol 2004 Apr;31(2 Suppl 6):30-6.

9. Dagan R, Morris CG, Zlotecki RA, Scarborough MT, Mendenhall WM. Radiotherapy in the treatment of dermatofibrosarcoma protuberans. Am J Clin Oncol 2005 Dec;28(6):537-9.

10. Paradisi A, Abeni D, Rusciani A et al. Dermatofibrosarcoma protuberans: wide local excision vs Mohs micrographic surgery. Cancer Treat Rev 2008 Dec;34(8):728-36. 